\title{
New crystallographic data and formula revision of phuralumite, $\mathrm{Al}_{2}\left[\left(\mathrm{UO}_{2}\right)_{3}\left(\mathrm{PO}_{4}\right)_{2} \mathrm{O}(\mathrm{OH})\right](\mathrm{OH})_{3}\left(\mathrm{H}_{2} \mathrm{O}\right)_{9}$
}

\author{
Fabrice DAL BO ${ }^{1}$, Frédéric HATERT ${ }^{1 *}$, Simon PHILIPPO² \\ ${ }^{1}$ Laboratoire de Minéralogie, B18, Université de Liège, B-4000Liège, Belgium; fhatert@ulg.ac.be \\ ${ }^{2}$ Section Minéralogie, Musée national d'histoire naturelle, Rue Münster 25, L-2160 Luxembourg, Grand-Duché de Luxembourg \\ * Corresponding author
}

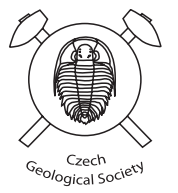

The crystal structure of phuralumite, $\mathrm{Al}_{2}\left[\left(\mathrm{UO}_{2}\right)_{3}\left(\mathrm{PO}_{4}\right)_{2} \mathrm{O}(\mathrm{OH})\right](\mathrm{OH})_{3}\left(\mathrm{H}_{2} \mathrm{O}\right)_{9}$, from the Kobokobo pegmatite (Kivu, Democratic Republic of Congo), was refined from single crystal X-ray diffraction data. Four samples have been investigated in this study, and the crystal structure was refined to $R_{1}=0.0566,0.0544,0.0661$ and 0.0353 , for 5739, 5953, 6007 and 5793 unique observed reflections, for the samples VC2048, VC2048, VC2054 and VC2055, respectively. Phuralumite is monoclinic, space group $P 2_{1} / n, a=9.4407(3), b=20.8596(8), c=13.4326(4) \AA, \beta=107.905(4)^{\circ}, V=2517.40(17)$ $\AA^{3}$, and $Z=4$ (sample VC2048). The structure consists of $\left[\left(\mathrm{UO}_{2}\right)_{3}\left(\mathrm{PO}_{4}\right)_{2} \mathrm{O}(\mathrm{OH})\right]^{3-}$ layers, parallel to $(010)$, which are connected by $\mathrm{Al}^{3+}$ ions and $\mathrm{H}_{2} \mathrm{O}$ molecules. The uranyl phosphate sheets show the phosphuranylite anion topology, while the $\mathrm{Al}^{3+}$ ions occur in 5- and 6-fold coordination and are connected together to form $\mathrm{Al}_{4} \mathrm{O}_{4}(\mathrm{OH})_{6}\left(\mathrm{H}_{2} \mathrm{O}\right)_{4}$ clusters. The crystallographic data obtained from the four structural models converge and confirm the previously determined structure for phuralumite. However, these new data also show some discrepancies in the bond-valence analysis, especially in the assignment of the $\mathrm{OH}^{-}$groups and water molecules. As a consequence of this study, the structural formula of phuralumite, previously reported as $\mathrm{Al}_{2}\left[\left(\mathrm{UO}_{2}\right)_{3}\left(\mathrm{PO}_{4}\right)_{2}(\mathrm{OH})_{2}\right](\mathrm{OH})_{4}\left(\mathrm{H}_{2} \mathrm{O}\right)_{10}$, must be modified to $\mathrm{Al}_{2}\left[\left(\mathrm{UO}_{2}\right)_{3}\left(\mathrm{PO}_{4}\right)_{2} \mathrm{O}(\mathrm{OH})\right](\mathrm{OH})_{3}\left(\mathrm{H}_{2} \mathrm{O}\right)_{9}$.

Keywords: phuralumite, crystal structure, aluminium, uranyl phosphate, Kobokobo, formula revision

Received: 20 December, 2016; accepted: 6 March, 2017; handling editor: J. Plášil

The online version of this article (doi: 10.3190/jgeosci.233) contains supplementary electronic material

\section{Introduction}

Uranyl phosphates and arsenates constitute the most diverse group of uranyl minerals, with more than eighty species described to date. The reason of this abundance is the wide distribution of phosphorus in many kinds of geological environments, and especially in the oxidation zones of uranium deposits (Finch and Murakami 1999; Krivovichev and Plášil 2013). One of the most remarkable occurrences of uranyl phosphates is the uraniferous quartz-albite-muscovite pegmatite of Kobokobo, situated in the region of Kivu in the western Democratic Republic of Congo (DRC). This pegmatite is the type-locality for twelve actinides- and aluminium-bearing phosphates: althupite, eylettersite, kamitugaite, metavanmeersscheite, moreautite, mundite, phuralumite, ranunculite, threadgoldite, triangulite, upalite and vanmeersscheite.

Phuralumite, $\mathrm{Al}_{2}\left[\left(\mathrm{UO}_{2}\right)_{3}\left(\mathrm{PO}_{4}\right)_{2}(\mathrm{OH})_{2}\right](\mathrm{OH})_{4}\left(\mathrm{H}_{2} \mathrm{O}\right)_{10}$, was originally described from the Kobokobo pegmatite by Deliens and Piret (1979), and is associated with meta-autunite, $\mathrm{Ca}\left[\left(\mathrm{UO}_{2}\right)\left(\mathrm{PO}_{4}\right)\right]_{2}\left(\mathrm{H}_{2} \mathrm{O}\right)_{6}$, phosphuranylite, $\mathrm{CaK}\left(\mathrm{H}_{3} \mathrm{O}\right)_{3}\left(\mathrm{UO}_{2}\right)\left[\left(\mathrm{UO}_{2}\right)_{3}\left(\mathrm{PO}_{4}\right)_{2} \mathrm{O}_{2}\right]_{2}\left(\mathrm{H}_{2} \mathrm{O}\right)_{8}$, and ranunculite, $\mathrm{Al}\left(\mathrm{UO}_{2}\right)\left(\mathrm{PO}_{3} \mathrm{OH}\right)(\mathrm{OH})_{3}\left(\mathrm{H}_{2} \mathrm{O}\right)_{4}$. Piret et al. (1979) solved the structure of phuralumite and confirmed the chemical composition previously obtained from electron-microprobe analyses by Deliens and Piret (1979).
Phuralumite is monoclinic, $P 2_{1} / a, Z=4, a=13.836(6)$, $b=20.918(6), c=9.428$ (3) $\AA, \beta=112.44(3)^{\circ}$. The structure is based on sheets showing the composition $\left[\left(\mathrm{UO}_{2}\right)_{3}\left(\mathrm{PO}_{4}\right)_{2}(\mathrm{OH})_{2}\right]^{2-}$, similar to those observed in the minerals of the phosphuranylite group (Krivovichev and Plášil 2013). These minerals are characterized by a U:P (or U:As) ratio of 3:2, and occur under slightly alkaline conditions $(\mathrm{pH} c .7 .5-8.5)$ in the end of the acidic stage of the development of oxidation zones (Chernikov 1981; Plášil et al. 2009; Krivovichev and Plášil 2013; Plášil 2014). Note that in addition to phosphates and arsenates, five uranyl selenites are structurally related to the phosphuranylite group: guilleminite $\mathrm{Ba}\left(\mathrm{UO}_{2}\right)_{3}\left(\mathrm{SeO}_{3}\right)_{2} \mathrm{O}_{2}\left(\mathrm{H}_{2} \mathrm{O}\right)_{3}$ (Cooper and Hawthorne 1995), piretite $\mathrm{Ca}\left(\mathrm{UO}_{2}\right)_{3}\left(\mathrm{SeO}_{3}\right)_{2}(\mathrm{OH})_{4}\left(\mathrm{H}_{2} \mathrm{O}\right)_{4}$ (Vochten et al. 1996), haynesite $\left(\mathrm{UO}_{2}\right)_{3}\left(\mathrm{SeO}_{3}\right)_{2}(\mathrm{OH})_{2}\left(\mathrm{H}_{2} \mathrm{O}\right)_{5}($ Čejka et al. 1999), marthozite $\mathrm{Cu}\left(\mathrm{UO}_{2}\right)_{3}\left(\mathrm{SeO}_{3}\right)_{2} \mathrm{O}_{2}\left(\mathrm{H}_{2} \mathrm{O}\right)_{8}$ (Cooper and Hawthorne 2001) and larisaite $\mathrm{Na}\left(\mathrm{H}_{3} \mathrm{O}\right)$ $\left(\mathrm{UO}_{2}\right)_{3}\left(\mathrm{SeO}_{3}\right)_{2} \mathrm{O}_{2}\left(\mathrm{H}_{2} \mathrm{O}\right)_{4}$ (Chukanov et al. 2004).

During our reinvestigation of uranyl minerals from the Kobokobo pegmatite, several samples of phuralumite have been studied (Dal Bo 2016). The new crystallographic data obtained on these samples show some discrepancy with those reported by Piret et al. (1979), and prompt us to publish new single-crystal X-ray diffraction data in order to revise the formula of phuralumite. 


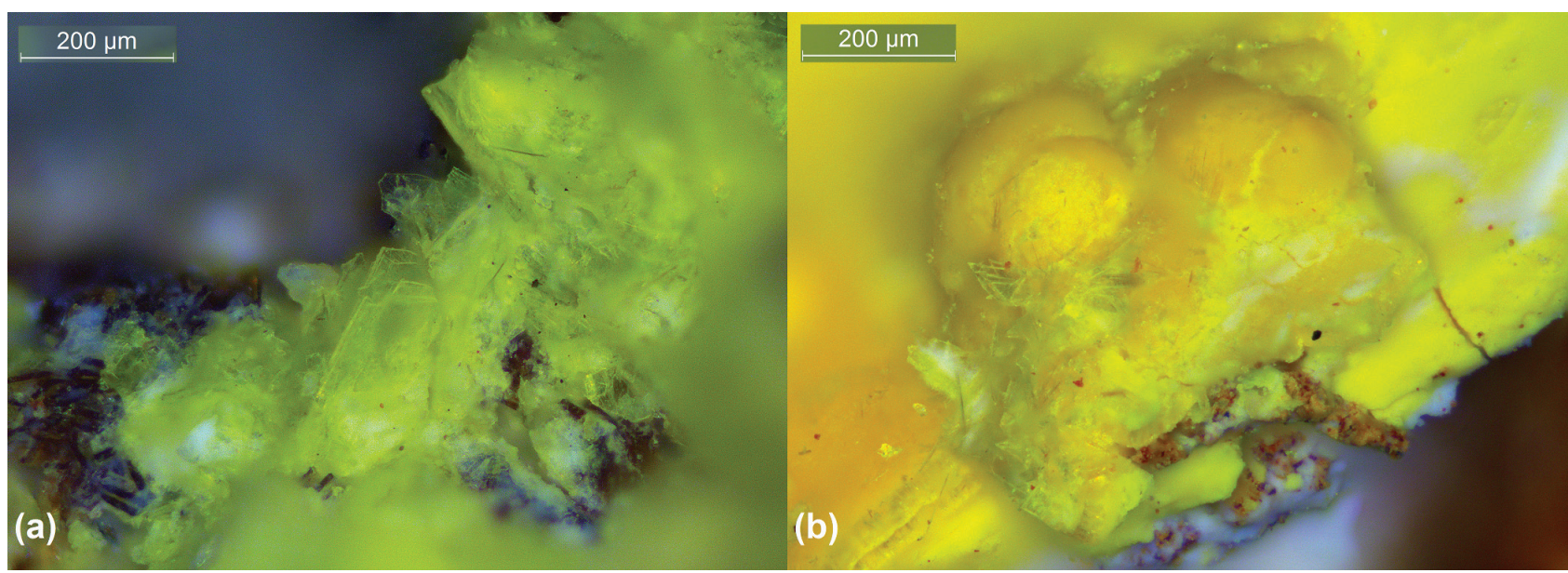

Fig. 1 Intergrowth of phuralumite prismatic crystals (a) and view of the close association between phuralumite and the nodules of ranunculite (b). Sample VC2055; photographs by R. Warin.

\section{Sample description}

Four samples from the Kobokobo pegmatite (VC2040, VC2048, VC2054 and VC2055) containing phuralumite crystals have been investigated. Phuralumite occurs as flattened and irregular monoclinic lemon-yellow prisms, commonly in subparallel or random intergrowths (Fig. 1). The crystals can reach up to $0.25 \mathrm{~mm}$ in length. In the present case, phuralumite is observed in close association with gold-yellow nodules of ranunculite. These samples belong to the collection of the Musée National d'Histoire Naturelle du Luxembourg.

\section{Chemical composition}

Energy-dispersive X-ray spectroscopy (EDS) was used to confirm the chemical composition of phuralumite. The

Tab. 1 Crystallographic data and refinement details for phuralumite samples

\begin{tabular}{|c|c|c|c|c|}
\hline Sample & VC2040 & VC2048 & VC2054 & VC2055 \\
\hline Ideal structural formula & \multicolumn{4}{|c|}{$\mathrm{Al}_{2}\left[\left(\mathrm{UO}_{2}\right)_{3}\left(\mathrm{PO}_{4}\right)_{2} \mathrm{O}(\mathrm{OH})\right](\mathrm{OH})_{3}\left(\mathrm{H}_{2} \mathrm{O}\right)_{9}$} \\
\hline$a(\AA)$ & $9.4280(3)$ & $9.4407(3)$ & $9.4423(5)$ & $9.4467(2)$ \\
\hline$b$ & $20.8445(5)$ & $20.8596(8)$ & $20.8723(9)$ & $20.9023(6)$ \\
\hline$c$ & $13.4207(4)$ & $13.4326(6)$ & $13.4307(6)$ & $13.4396(3)$ \\
\hline$\beta\left(^{\circ}\right)$ & $107.962(3)$ & $107.905(4)$ & $107.862(5)$ & 107.944(3) \\
\hline$V\left(\AA^{3}\right)$ & $2508.92(12)$ & $2517.40(17)$ & $2519.3(5)$ & $2524.68(11)$ \\
\hline Space group & $P 2_{1} / n$ & $P 2_{1} / n$ & $P 2_{1} / n$ & $P 2_{1} / n$ \\
\hline$Z$ & 4 & 4 & 4 & 4 \\
\hline Calculated density $\left(\mathrm{g} \cdot \mathrm{cm}^{-3}\right)$ & 3.394 & 3.383 & 3.380 & 3.373 \\
\hline Absorption coefficient $\left(\mathrm{mm}^{-1}\right)$ & 19.621 & 19.555 & 19.540 & 19.498 \\
\hline$F(000)$ & 2272 & 2408 & 2272 & 2272 \\
\hline Radiation $(\AA)$ & $\operatorname{Mo} K_{\alpha}, 0.71073$ & $\operatorname{Mo} K_{\alpha}, 0.71073$ & $\operatorname{Mo} K_{\alpha}, 0.71073$ & $\operatorname{Mo} K_{\alpha}, 0.71073$ \\
\hline Crystal size (mm) & $0.12 \times 0.11 \times 0.08$ & $0.13 \times 0.07 \times 0.03$ & $0.16 \times 0.13 \times 0.06$ & $0.19 \times 0.04 \times 0.02$ \\
\hline Temperature $(\mathrm{K})$ & 293 & 293 & 293 & 293 \\
\hline \multirow[t]{2}{*}{$\theta$ range $\left(^{\circ}\right)$} & 2.33 to 28.47 & 2.32 to 28.18 & 2.34 to 27.21 & 2.45 to 28.84 \\
\hline & $-12 \leq h \leq 11$ & $-12 \leq h \leq 12$ & $-12 \leq h \leq 12$ & $-12 \leq h \leq 12$ \\
\hline \multirow[t]{2}{*}{ Reflection range } & $-28 \leq k \leq 25$ & $-27 \leq k \leq 27$ & $-28 \leq k \leq 27$ & $-27 \leq k \leq 26$ \\
\hline & $-17 \leq l \leq 14$ & $-17 \leq l \leq 16$ & $-17 \leq l \leq 17$ & $-17 \leq l \leq 17$ \\
\hline Total no. of reflections & 11860 & 19906 & 23278 & 13308 \\
\hline Unique reflections & 5739 & 5953 & 6007 & 5793 \\
\hline Observed reflections, $\left|F_{\mathrm{O}}\right| \geq 4 \sigma F$ & 4268 & 3911 & 3937 & 4665 \\
\hline Refined parameters & 314 & 314 & 304 & 329 \\
\hline$R_{1},\left|F_{\mathrm{O}}\right| \geq 4 \sigma F$ & 0.0566 & 0.0544 & 0.0661 & 0.0353 \\
\hline$R_{1}$, all data & 0.0831 & 0.1035 & 0.1148 & 0.0522 \\
\hline$w R_{2}\left(F^{2}\right)$, all data & 0.1454 & 0.1379 & 0.1655 & 0.0835 \\
\hline GOF obs/all & $1.07,1.07$ & $1.04,1.04$ & $1.04,1.04$ & $1.02,1.02$ \\
\hline$\Delta \rho_{\min }, \Delta \rho_{\max }\left(\mathrm{e} / \AA^{3}\right)$ & $5.00,-2.64$ & $2.64,-2.27$ & $3.37,-3.95$ & $2.08,-2.06$ \\
\hline
\end{tabular}


crystals were mounted on adhesive carbon tape, carbon-coated, and then analysed by an environmental scanning electron microscope (FEX XL30 ESEMFEG) working in high vacuum mode, using $15 \mathrm{kV}$ accelerating potential. In all analysed crystals the only chemical elements detected are U, P, Al and O (Fig. 2). Samples VC2048, VC2054 and VC2055 also revealed a small amount of $\mathrm{Si}$; however, the presence of this element in the crystal structure of phuralumite is not supported by the crystallographic data. The absence of As is consistent with the geological environment (pegmatite) in which this element is usually very scarce. Presence of fluorine was carefully checked and this chemical element remains absent. No other elements, especially those prone to substitute for $\mathrm{Al}$, were detected.

\section{X-ray crystallography} and structure determination

\subsection{Single-crystal $X$-ray diffraction and structure solution}

Single-crystal X-ray study of phuralumite was carried out with an Rigaku Xcalibur fourcircle diffractometer (kappa geometry), using $\mathrm{Mo} K_{\alpha}$ radiation $(\lambda=0.71073 \AA, 40 \mathrm{kV}$, $40 \mathrm{~mA}$ ), and equipped with an EOS CCD area detector, on crystal fragments from four different samples VC2040, VC2048, VC2054 and VC2055.

Fig. 2 Energy-dispersive X-ray emission spectra of furongite. The labelled peaks correspond to the following lines: $\mathrm{a}-\mathrm{C} K_{\alpha} ; \mathrm{b}-\mathrm{O} K_{\alpha} ; \mathrm{c}-\mathrm{A} 1 K_{\alpha} ; \mathrm{d}-\mathrm{Si} K_{\alpha} ;$ $\mathrm{e}-\mathrm{P} K_{\alpha} ; \mathrm{f}-\mathrm{U} M_{\zeta} ; \mathrm{g}-\mathrm{U} M_{\alpha} ; \mathrm{h}-\mathrm{U} M_{\beta}$; $\mathrm{i}-\mathrm{U} M_{\gamma}$.
.
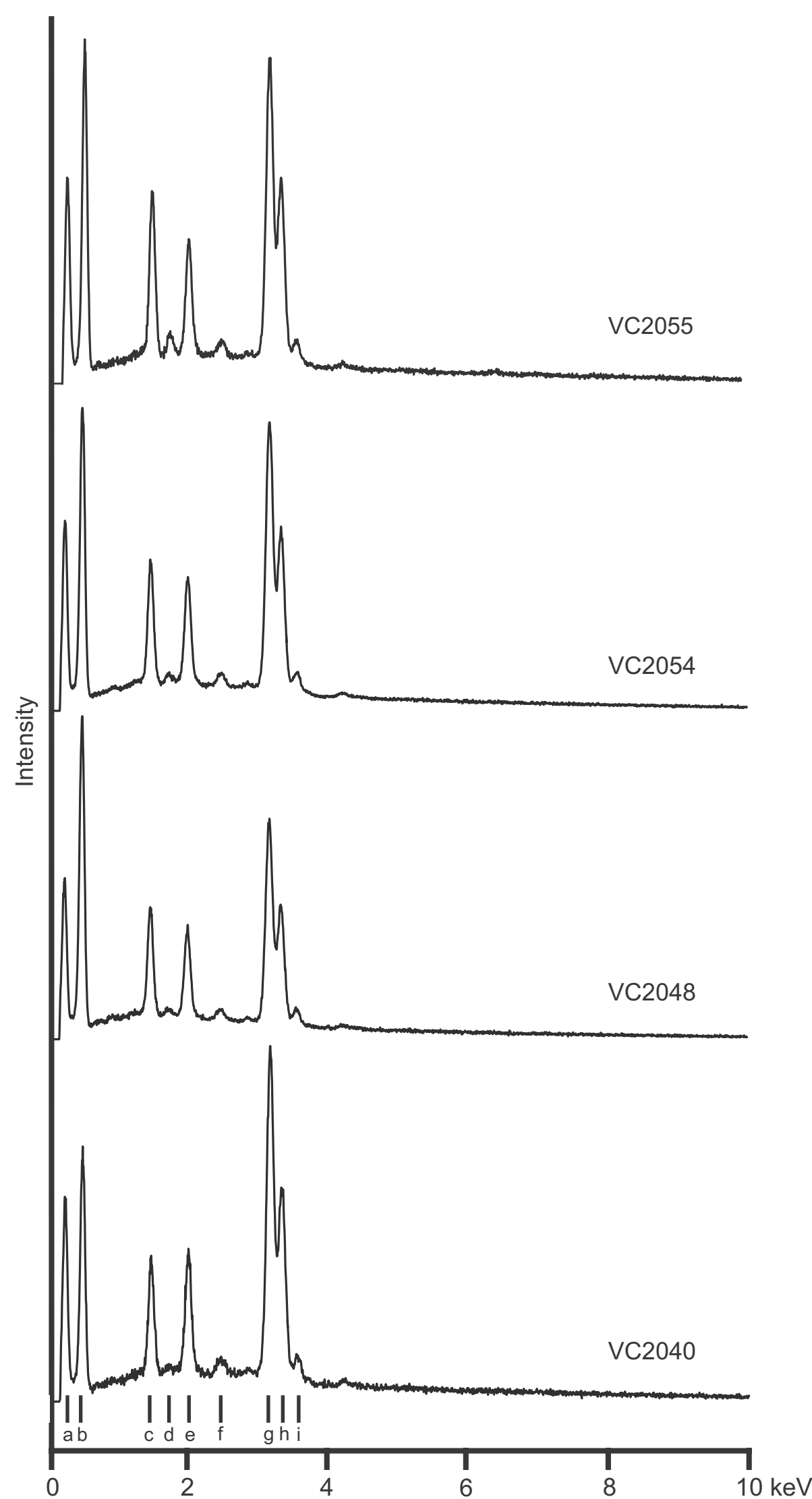


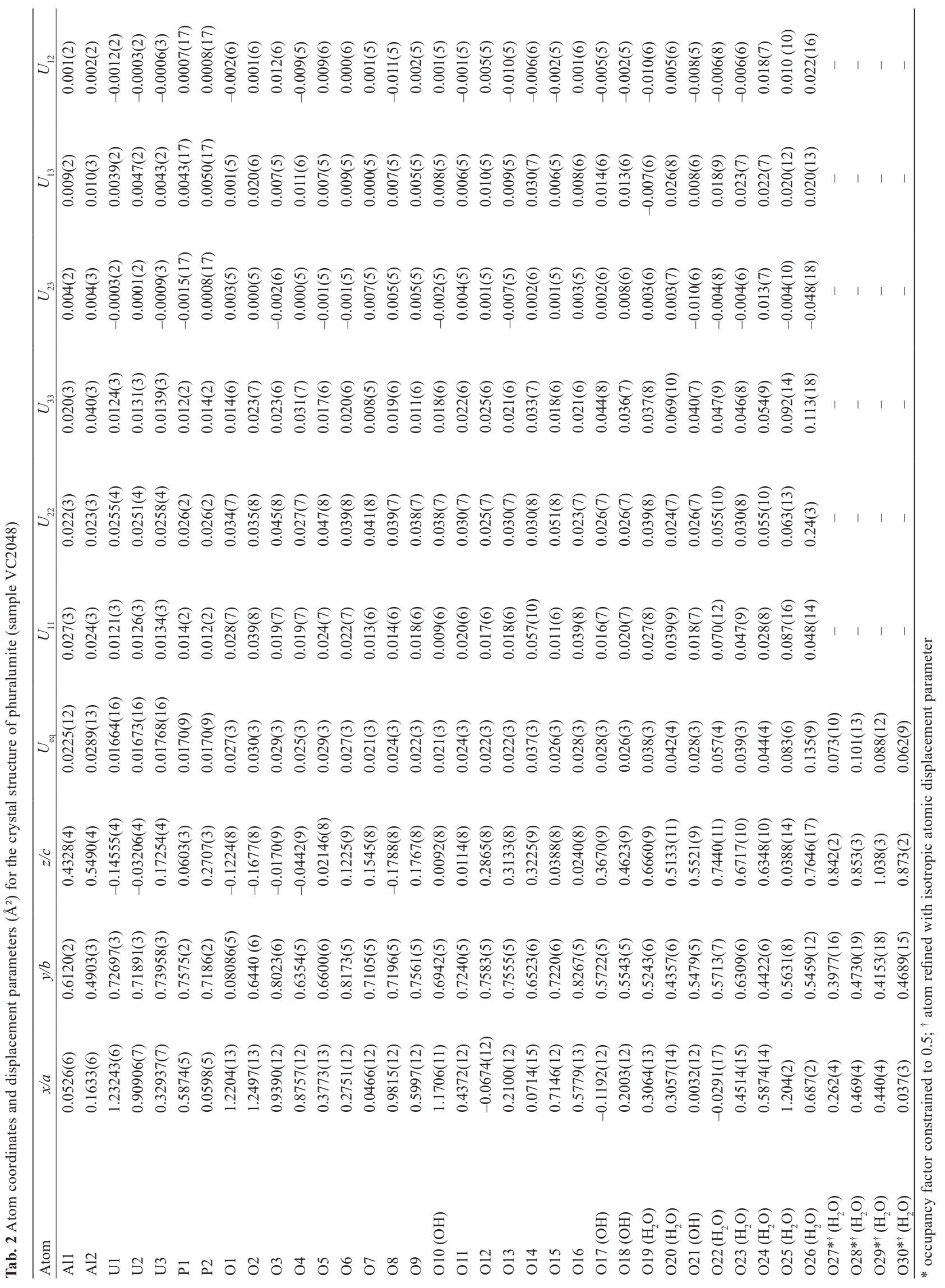


Tab.3 Selected interatomic in the structure of phuralumite

\begin{tabular}{|c|c|c|c|c|}
\hline & VC2040 & VC2048 & VC2054 & VC2055 \\
\hline U1-O1 & $1.740(12)$ & $1.740(11)$ & $1.751(13)$ & $1.759(7)$ \\
\hline $\mathrm{U} 1-\mathrm{O} 2$ & $1.770(10)$ & $1.772(12)$ & $1.757(11)$ & $1.765(7)$ \\
\hline U1-O8 & $2.282(9)$ & $2.277(10)$ & $2.267(13)$ & $2.273(5)$ \\
\hline U1-O9 & $2.330(10)$ & $2.357(10)$ & $2.356(11)$ & $2.360(6)$ \\
\hline $\mathrm{U} 1-\mathrm{O} 10$ & $2.420(9)$ & $2.425(9)$ & $2.409(12)$ & $2.416(6)$ \\
\hline $\mathrm{U} 1-\mathrm{O} 11$ & $2.377(11)$ & $2.383(11)$ & $2.395(11)$ & $2.380(6)$ \\
\hline $\mathrm{U} 1-\mathrm{O} 12$ & $2.341(10)$ & $2.358(10)$ & $2.345(14)$ & $2.355(6)$ \\
\hline$<\mathrm{U} 1-\mathrm{O}_{U r}>$ & 1.75 & 1.76 & 1.75 & 1.76 \\
\hline$<\mathrm{U} 1-\mathrm{O}_{e q}>$ & 2.35 & 2.36 & 2.35 & 2.36 \\
\hline $\mathrm{U} 2-\mathrm{O} 3$ & $1.809(12)$ & $1.765(12)$ & $1.772(12)$ & $1.777(7)$ \\
\hline U2-O4 & $1.771(11)$ & $1.769(11)$ & $1.749(12)$ & $1.787(7)$ \\
\hline U2-O7 & $2.451(11)$ & $2.449(10)$ & $2.464(12)$ & $2.458(6)$ \\
\hline U2-O8 & $2.264(9)$ & $2.277(10)$ & $2.301(13)$ & $2.260(5)$ \\
\hline $\mathrm{U} 2-\mathrm{O} 10$ & $2.406(10)$ & $2.414(10)$ & $2.425(12)$ & $2.416(6)$ \\
\hline $\mathrm{U} 2-\mathrm{O} 13$ & $2.396(10)$ & $2.393(11)$ & $2.410(11)$ & $2.399(6)$ \\
\hline $\mathrm{U} 2-\mathrm{O} 15$ & $2.325(10)$ & $2.314(10)$ & $2.340(15)$ & $2.328(6)$ \\
\hline$<\mathrm{U} 2-\mathrm{O}_{U r}>$ & 1.79 & 1.77 & 1.76 & 1.78 \\
\hline$<\mathrm{U} 2-\mathrm{O}_{e q}>$ & 2.37 & 2.37 & 2.39 & 2.37 \\
\hline U3-O5 & $1.780(11)$ & $1.767(12)$ & $1.768(12)$ & $1.767(7)$ \\
\hline U3-O6 & $1.780(11)$ & $1.769(11)$ & $1.760(12)$ & $1.773(7)$ \\
\hline $\mathrm{U} 3-\mathrm{O} 7$ & $2.662(10)$ & $2.675(10)$ & $2.686(14)$ & $2.668(6)$ \\
\hline U3-O8 & $2.225(10)$ & $2.238(11)$ & $2.223(11)$ & $2.246(6)$ \\
\hline U3-O9 & $2.559(9)$ & $2.559(10)$ & $2.556(13)$ & $2.555(6)$ \\
\hline $\mathrm{U} 3-\mathrm{O} 10$ & $2.432(10)$ & $2.436(11)$ & $2.412(11)$ & $2.430(6)$ \\
\hline U3-O11 & $2.693(10)$ & $2.680(10)$ & $2.665(13)$ & $2.685(6)$ \\
\hline U3-O13 & $2.523(10)$ & $2.505(9)$ & $2.532(13)$ & $2.505(6)$ \\
\hline$<\mathrm{U} 3-\mathrm{O}_{U r}>$ & 1.78 & 1.77 & 1.76 & 1.77 \\
\hline$<\mathrm{U} 3-\mathrm{O}_{e q}>$ & 2.52 & 2.52 & 2.51 & 2.51 \\
\hline P1-O9 & $1.560(10)$ & $1.533(10)$ & $1.542(12)$ & $1.525(6)$ \\
\hline $\mathrm{P} 1-\mathrm{O} 11$ & $1.509(10)$ & $1.536(12)$ & $1.519(13)$ & $1.532(7)$ \\
\hline $\mathrm{P} 1-\mathrm{O} 15$ & $1.496(12)$ & $1.512(11)$ & $1.486(14)$ & $1.508(7)$ \\
\hline $\mathrm{P} 1-\mathrm{O} 16$ & $1.516(11)$ & $1.518(12)$ & $1.497(12)$ & $1.516(7)$ \\
\hline$<\mathrm{P} 1-\mathrm{O}>$ & 1.52 & 1.52 & 1.51 & 1.52 \\
\hline $\mathrm{P} 2-\mathrm{O} 7$ & $1.532(11)$ & $1.536(10)$ & $1.531(12)$ & $1.531(6)$ \\
\hline $\mathrm{P} 2-\mathrm{O} 12$ & $1.531(11)$ & $1.526(11)$ & $1.523(14)$ & $1.521(6)$ \\
\hline $\mathrm{P} 2-\mathrm{O} 13$ & $1.533(10)$ & $1.560(11)$ & $1.535(13)$ & $1.549(6)$ \\
\hline $\mathrm{P} 2-\mathrm{O} 14$ & $1.527(10)$ & $1.536(12)$ & $1.513(12)$ & $1.527(7)$ \\
\hline$<\mathrm{P} 2-\mathrm{O}>$ & 1.53 & 1.54 & 1.53 & 1.53 \\
\hline $\mathrm{A} 11-\mathrm{O} 14$ & $1.778(11)$ & $1.760(12)$ & $1.795(13)$ & $1.782(7)$ \\
\hline Al1-O16 & $1.736(11)$ & $1.735(12)$ & $1.756(12)$ & $1.745(7)$ \\
\hline Al1-O17 & $1.780(11)$ & $1.795(12)$ & $1.793(14)$ & $1.788(7)$ \\
\hline Al1-O18 & $1.766(11)$ & $1.792(12)$ & $1.771(14)$ & $1.786(7)$ \\
\hline $\mathrm{A} 11-\mathrm{O} 21$ & $2.205(11)$ & $2.244(12)$ & $2.238(14)$ & $2.195(7)$ \\
\hline$<\mathrm{All}-\mathrm{O}>$ & 1.85 & 1.86 & 1.87 & 1.86 \\
\hline $\mathrm{A} 12-\mathrm{O} 17$ & $1.860(11)$ & $1.854(12)$ & $1.855(13)$ & $1.855(7)$ \\
\hline A12-O18 & $1.876(11)$ & $1.872(11)$ & $1.879(14)$ & $1.866(7)$ \\
\hline A12-O19 & $1.858(12)$ & $1.869(13)$ & $1.875(14)$ & $1.884(8)$ \\
\hline $\mathrm{A} 12-\mathrm{O} 20$ & $1.914(12)$ & $1.932(13)$ & $1.928(15)$ & $1.937(8)$ \\
\hline $\mathrm{A} 12-\mathrm{O} 21$ & $1.913(12)$ & $1.907(12)$ & $1.917(13)$ & $1.920(7)$ \\
\hline $\mathrm{A} 12-\mathrm{O} 21^{\mathrm{i}}$ & $1.945(11)$ & $1.941(12)$ & $1.931(14)$ & $1.946(7)$ \\
\hline$<\mathrm{Al} 2-\mathrm{O}>$ & 1.89 & 1.90 & 1.90 & 1.90 \\
\hline
\end{tabular}

Symmetry code: (i) $-\mathrm{x} ; 1-\mathrm{y} ; 1-\mathrm{z}$
Tab. 4 The $\mathrm{O} \cdots \mathrm{O}$ separation distances found in the structure of phuralumite (sample VC2048)

\begin{tabular}{|c|c|}
\hline $\mathrm{O} 1-\mathrm{OH} 17^{\mathrm{i}}$ & $2.94(2)$ \\
\hline $\mathrm{O} 1-\mathrm{H}_{2} \mathrm{O} 20^{\mathrm{ii}}$ & $3.07(2)$ \\
\hline $\mathrm{O} 2-\mathrm{H}_{2}^{2} \mathrm{O} 22^{\mathrm{iii}}$ & $2.95(2)$ \\
\hline $\mathrm{O} 2-\mathrm{H}_{2} \mathrm{O} 23^{\mathrm{iii}}$ & $3.29(1)$ \\
\hline $\mathrm{O} 2-\mathrm{H}_{2} \mathrm{O} 29^{\text {iv }}$ & $3.18(4)$ \\
\hline $\mathrm{O} 3-\mathrm{H}_{2} \mathrm{O} 23^{\mathrm{v}}$ & $2.87(2)$ \\
\hline $\mathrm{O} 3-\mathrm{H}_{2} \mathrm{O} 24^{\mathrm{ii}}$ & $3.30(2)$ \\
\hline $\mathrm{O} 4-\mathrm{H}_{2}^{2} \mathrm{O} 26^{\mathrm{vii}}$ & $3.21(1)$ \\
\hline $\mathrm{O} 4-\mathrm{H}_{2} \mathrm{O} 29^{\mathrm{vi}}$ & $3.18(5)$ \\
\hline $\mathrm{O} 4-\mathrm{H}_{2} \mathrm{O} 30^{\mathrm{vi}}$ & $3.10(3)$ \\
\hline $\mathrm{O} 5-\mathrm{H}_{2} \mathrm{O} 24^{\mathrm{vi}}$ & $2.88(2)$ \\
\hline $\mathrm{O} 5-\mathrm{H}_{2} \mathrm{O} 25^{\text {viii }}$ & $3.15(2)$ \\
\hline $\mathrm{O} 6-\mathrm{H}_{2}^{2} \mathrm{O} 20^{\mathrm{ix}}$ & $3.02(2)$ \\
\hline $\mathrm{O} 6-\mathrm{H}_{2}^{2} \mathrm{O} 22^{v}$ & $3.10(2)$ \\
\hline $\mathrm{O} 8-\mathrm{H}_{2} \mathrm{O} 22^{\mathrm{iii}}$ & $3.26(2)$ \\
\hline $\mathrm{OH} 10-\mathrm{H}_{2} \mathrm{O} 25$ & $2.77(2)$ \\
\hline $\mathrm{O} 11-\mathrm{H}_{2} \mathrm{O} 29^{\mathrm{vi}}$ & $3.28(4)$ \\
\hline $\mathrm{O} 12-\mathrm{H}_{2}^{2} \mathrm{O} 23^{x}$ & $2.81(2)$ \\
\hline O14-OH17 & $2.65(2)$ \\
\hline O14-OH18 & $2.79(2)$ \\
\hline $\mathrm{O} 15-\mathrm{H}_{2} \mathrm{O} 27^{\mathrm{vi}}$ & $2.95(4)$ \\
\hline $\mathrm{O} 15-\mathrm{H}_{2} \mathrm{O} 29^{\mathrm{vi}}$ & $3.24(4)$ \\
\hline $\mathrm{O} 16-\mathrm{OH} 18^{\mathrm{v}}$ & $2.96(1)$ \\
\hline $\mathrm{O} 16-\mathrm{OH} 21^{\mathrm{v}}$ & $2.76(1)$ \\
\hline OH17-OH18 & 2.91(1) \\
\hline $\mathrm{OH} 17-\mathrm{H}_{2} \mathrm{O} 19^{\mathrm{xi}}$ & $2.63(2)$ \\
\hline $\mathrm{OH} 17-\mathrm{H}_{2} \mathrm{O} 20^{\mathrm{xi}}$ & $2.73(1)$ \\
\hline $\mathrm{OH} 17-\mathrm{OH} 21^{\mathrm{xi}}$ & $2.81(1)$ \\
\hline $\mathrm{OH} 17-\mathrm{OH} 21$ & $2.45(2)$ \\
\hline $\mathrm{OH} 17-\mathrm{H}_{2} \mathrm{O} 27^{\mathrm{xi}}$ & $2.76(3)$ \\
\hline $\mathrm{OH} 18-\mathrm{H}_{2}^{2} \mathrm{O} 19$ & $2.69(2)$ \\
\hline $\mathrm{OH} 18-\mathrm{H}_{2}^{2} \mathrm{O} 20$ & $2.68(2)$ \\
\hline OH18-OH21 & $2.51(1)$ \\
\hline $\mathrm{OH} 18-\mathrm{OH} 21^{\mathrm{xi}}$ & $2.83(2)$ \\
\hline $\mathrm{OH} 18-\mathrm{H}_{2} \mathrm{O} 24^{\mathrm{vi}}$ & $2.71(1)$ \\
\hline $\mathrm{OH}_{2} 19-\mathrm{H}_{2} \mathrm{O} 20$ & $2.77(8)$ \\
\hline $\mathrm{OH}_{2} 19-\mathrm{OH} 21$ & $2.84(1)$ \\
\hline $\mathrm{OH}_{2} 19-\mathrm{H}_{2} \mathrm{O} 23$ & $2.60(2)$ \\
\hline $\mathrm{OH}_{2} 19-\mathrm{H}_{2} \mathrm{O} 24$ & $3.29(2)$ \\
\hline $\mathrm{OH}_{2} 19-\mathrm{H}_{2}^{2} \mathrm{O} 28$ & $2.70(4)$ \\
\hline $\mathrm{OH}_{2} 20-\mathrm{OH} 21^{\mathrm{xi}}$ & $2.79(2)$ \\
\hline $\mathrm{OH}_{2} 20-\mathrm{H}_{2} \mathrm{O} 24$ & $2.68(1)$ \\
\hline $\mathrm{OH} 21-\mathrm{OH} 21^{\mathrm{xi}}$ & $2.42(1)$ \\
\hline $\mathrm{OH} 21-\mathrm{H}_{2} \mathrm{O} 22$ & $2.73(2)$ \\
\hline $\mathrm{OH} 22-\mathrm{H}_{2} \mathrm{O} 26^{\text {viii }}$ & $2.82(4)$ \\
\hline $\mathrm{OH}_{2} 22-\mathrm{H}_{2} \mathrm{O} 30$ & $2.69(3)$ \\
\hline $\mathrm{OH}_{2}^{2} 23-\mathrm{H}_{2}^{2} \mathrm{O} 26$ & $2.78(3)$ \\
\hline $\mathrm{OH}_{2} 24-\mathrm{H}_{2} \mathrm{O} 26$ & $2.79(3)$ \\
\hline $\mathrm{OH}_{2} 25-\mathrm{H}_{2} \mathrm{O} 28^{\mathrm{iv}}$ & $3.09(4)$ \\
\hline $\mathrm{OH}_{2} 25-\mathrm{H}_{2} \mathrm{O} 30^{\mathrm{vi}}$ & $2.93(4)$ \\
\hline $\mathrm{OH}_{2} 25-\mathrm{H}_{2} \mathrm{O} 30^{\mathrm{xii}}$ & $3.07(3)$ \\
\hline $\mathrm{OH}_{2} 26-\mathrm{H}_{2} \mathrm{O} 28^{\mathrm{iv}}$ & $3.10(5)$ \\
\hline $\mathrm{OH}_{2}^{2} 27-\mathrm{H}_{2}^{2} \mathrm{O} 28$ & $2.48(6)$ \\
\hline $\mathrm{OH}_{2} 27-\mathrm{H}_{2} \mathrm{O} 29$ & $2.69(5)$ \\
\hline $\mathrm{OH}_{2} 27-\mathrm{H}_{2} \mathrm{O} 30$ & $2.74(5)$ \\
\hline $\mathrm{OH}_{2} 28-\mathrm{H}_{2} \mathrm{O} 29^{\mathrm{xiii}}$ & $2.76(6)$ \\
\hline $\mathrm{OH}_{2}^{2} 28-\mathrm{H}_{2}^{2} \mathrm{O} 29$ & $2.86(5)$ \\
\hline
\end{tabular}

Symmetry codes: (i) $3 / 2+x, 3 / 2-y,-1 / 2+z$; (ii) $3 / 2-x, 1 / 2+y, 1 / 2-z$; (iii) $1+\mathrm{x}, \mathrm{y},-1+\mathrm{z}$; (iv) $2-\mathrm{x}, 1-\mathrm{y}, 1-\mathrm{z}$; (v) $1 / 2+\mathrm{x}, 3 / 2-\mathrm{y},-1 / 2+\mathrm{z}$; (vi) $1-\mathrm{x}$, $1-\mathrm{y}, 1-\mathrm{z}$; (vii) $\mathrm{x}, \mathrm{y},-1+\mathrm{z}$; (viii) $-1+\mathrm{x}, \mathrm{y}, \mathrm{z}$; (ix) $1 / 2-\mathrm{x}, 1 / 2+\mathrm{y}, 1 / 2-\mathrm{z}$; (x) $-1 / 2+x, 3 / 2-y,-1 / 2+z$; (xi) $-\mathrm{x}, 1-\mathrm{y}, 1-\mathrm{z}$; (xii) $-1+\mathrm{x}, \mathrm{y}, 1+\mathrm{z}$; (xiii) $1-\mathrm{x}, 1-\mathrm{y}, 2-\mathrm{z}$ 
The crystallographic data and the refinement details on these phuralumite crystals are reported in the Tab. 1. The data were corrected for Lorentz, polarization and absorption effects, the latter with an empirical method using the SCALE3 ABSPACK scaling algorithm included in the CrysAlisRED software package (Agilent Technologies 2012). The crystal structures were solved by direct methods and subsequently refined using SHELXS and SHELXL software (Sheldrick 2008). The reflection conditions and statistics clearly indicate the $P 2_{1} / n$ space group, consistent with the $P 2{ }_{1} / a$ space group previously reported by Piret et al. (1979) (transformation matrix [101/010/-100]). Scattering curves for neutral atoms and anomalous dispersion correction were taken from Wilson (1992).

The structure of phuralumite was solved by direct methods and was refined successfully on the basis of $F^{2}$ for all unique data in space group $P 2_{1} / n$. Structure models including anisotropic displacement parameters for all non- $\mathrm{H}$ atoms converged and gave an agreement index $(R 1)$ of $5.66 \%, 5.44 \%, 6.61 \%$ and $3.53 \%$ calculated for the 4268, 3911, 3937 and 4665 observed unique reflections $\left(\left|F_{\mathrm{o}}\right| \geq 4 \sigma_{F}\right)$ (VC2040, VC2048, VC2054 and $\mathrm{VC} 2055$, respectively). The relative occupancy of the $\mathrm{Al}$ and $\mathrm{P}$ sites was freely refined in order to detect some cationic substitutions. The relative occupancies of all $\mathrm{Al}$ and P sites stay very close to unity, and therefore these $\mathrm{Al}$ and $\mathrm{P}$ sites were considered as fully occupied. This observation is consistent with the cationic content of the phuralumite samples VC2040, as confirmed by EDS analyses. The presence of $\mathrm{Si}$ in phuralumite samples VC2048, VC2054 and VC2055 is not supported by the relative occupancy of the $\mathrm{P}$ sites. The occupancy factor of the $\mathrm{O} 27$ to $\mathrm{O} 30$ sites, which are occupied by water molecules, has been set to 0.5 in order to keep their isotropic atom displacement parameters at acceptable values. Final atom coordinates, isotropic atom displacement parameters and site occupancies, are given in Tab. 2 for sample VC2048, and selected interatomic distances are reported in Tabs 3 and 4. Bond-valence sums (BVS) were calculated with the parameters of Brown and Altermatt (1985) for $\mathrm{Al}$ and $\mathrm{P}$, and those of Burns et al. (1997) for $\mathrm{U}^{6+}$ (Tab. 5). Final atom coordinates, as well as the bond-valence tables for samples VC2040, VC2054 and VC2055, are provided as electronic supplementary materials $1-6$.

\subsection{Description of the crystal structure}

The crystal structure of phuralumite contains three symmetrically independent $\mathrm{U}^{6+}$ cations which are forming typical nearly linear $\mathrm{UO}_{2}{ }^{2+}$ uranyl ions $(U r)$. $\mathrm{U} 1$ and $\mathrm{U} 2$ are coordinated by five additional oxygen atoms located at the equatorial vertices of the uranyl ions to form the $U r \mathrm{O}_{4}(\mathrm{OH})$ pentagonal bipyramids. The $\left\langle\mathrm{U}-\mathrm{O}_{U r}>\right.$ and $<\mathrm{U}-\mathrm{O}_{e q}>(e q=$ equatorial $)$ mean

Fig. 3 General view of the structure of phuralumite (a) and view of one isolated uranyl phosphate sheet running parallel to (010) (b). $\mathrm{UO}_{7}$ are yellow, $\mathrm{UO}_{8}$ orange, Al octahedra blue, hydroxyl groups grey and oxygen atoms and water molecules red. Unit-cell edges are outlined by the solid black line. (VESTA 3 software; Momma and Izumi 2011). 
bond lengths vary between 1.76 and $1.79 \AA$, and between 2.35 and $2.39 \AA$, respectively (Tab. $3)$. U3 is coordinated by six additional oxygen atoms located at the equatorial vertices of the uranyl ion to form $U r \mathrm{O}_{5}(\mathrm{OH})$ hexagonal bipyramids. The $<\mathrm{U}-$ $\mathrm{O}_{U r}>$ and $\left\langle\mathrm{U}-\mathrm{O}_{e q}>\right.$ mean bond lengths are 1.77-1.78 $\AA$, and $2.51 \AA$, respectively. These values closely match the typical bond lengths reported by Burns et al. (1997) for $\mathrm{UO}_{7}$ and $\mathrm{UO}_{8}$ polyhedra. Two $\mathrm{P}^{5+}$ cations are also occurring in the structure as $\mathrm{PO}_{4}$ tetrahedra. The $<\mathrm{P}-\mathrm{O}>$ mean bond lengths vary between 1.51 and $1.54 \AA$. The $\mathrm{UrO}_{5}$ pentagonal bipyramids formed dimer by edge-sharing, which are then sharing edges with $U r \mathrm{O}_{6}$ hexagonal bipyramids to form chains. Adjacent chains are connected together by corner- and edge-sharing $\mathrm{PO}_{4}$ tetrahedra, yielding a $\left[\left(\mathrm{UO}_{2}\right)_{3}\left(\mathrm{PO}_{4}\right)_{2} \mathrm{O}(\mathrm{OH})\right]^{3-}$ sheet parallel to (101) (Fig. 3). Two independent $\mathrm{Al}^{3+}$ cations are occurring in 5-fold coordination (Al1) and in octahedral coordination (A12). Al1 form a trigonal bipyramid characterized by four $\mathrm{Al}-\phi\left(\phi=\mathrm{O}^{2-}, \mathrm{OH}^{-}\right.$and $\left.\mathrm{H}_{2} \mathrm{O}\right)$ bond lengths of 1.76-1.77 $\AA$, and one longer $\mathrm{Al}-\phi$ bond length of 2.20-2.24 A. Four $\mathrm{AllO}_{2}(\mathrm{OH})_{3}$ and $\mathrm{Al} 2(\mathrm{OH})_{4}\left(\mathrm{H}_{2} \mathrm{O}\right)_{2}$ are connected by edge-sharing to form $\mathrm{Al}_{4} \mathrm{O}_{4}(\mathrm{OH})_{6}\left(\mathrm{H}_{2} \mathrm{O}\right)_{4}$ clusters, which are linked to free apical oxygen atoms of the $\mathrm{PO}_{4}$ tetrahedra (Fig. 4). Finally, the interlayer of the structure contains eight isolated water molecules, four of which have a site occupation constrained to 0.5. The structural formula of phuralumite obtained from the refinement is $\mathrm{Al}_{2}\left[\left(\mathrm{UO}_{2}\right)_{3}\left(\mathrm{PO}_{4}\right)_{2} \mathrm{O}(\mathrm{OH})\right](\mathrm{OH})_{3}\left(\mathrm{H}_{2} \mathrm{O}\right)_{9}$.

\section{Discussion}

The general features of the phuralumite structure reported in this work are identical to those observed by Piret et al. (1979). The sheets of uranyl polyhedra and phosphate tetrahedra show the phosphuranylite anion topology, characterized by triangles, squares, pentagons and hexagons (Burns 2005). However, close examination
Tab. 5 The bond-valence analysis for phuralumite (sample VC2048)

\begin{tabular}{|c|c|c|c|c|c|c|c|c|c|}
\hline & Al1 & $\mathrm{A} 12$ & U1 & U2 & U3 & P1 & $\mathrm{P} 2$ & $\Sigma$ & Species \\
\hline $\mathrm{O} 1$ & & & 1.819 & & & & & 1.82 & $\mathrm{O}$ \\
\hline $\mathrm{O} 2$ & & & 1.708 & & & & & 1.71 & $\mathrm{O}$ \\
\hline $\mathrm{O} 3$ & & & & 1.732 & & & & 1.73 & $\mathrm{O}$ \\
\hline $\mathrm{O} 4$ & & & & 1.718 & & & & 1.72 & $\mathrm{O}$ \\
\hline O5 & & & & & 1.722 & & & 1.72 & $\mathrm{O}$ \\
\hline O6 & & & & & 1.715 & & & 1.72 & $\mathrm{O}$ \\
\hline O7 & & & & 0.453 & 0.286 & & 1.245 & 1.98 & $\mathrm{O}$ \\
\hline O8 & & & 0.635 & 0.635 & 0.679 & & & 1.95 & $\mathrm{O}$ \\
\hline O9 & & & 0.542 & & 0.360 & 1.255 & & 2.16 & $\mathrm{O}$ \\
\hline $\mathrm{O} 10$ & & & 0.475 & 0.485 & 0.459 & & & 1.42 & $\mathrm{OH}$ \\
\hline O11 & & & 0.515 & & 0.283 & 1.245 & & 2.04 & $\mathrm{O}$ \\
\hline $\mathrm{O} 12$ & & & 0.541 & & & & 1.279 & 1.82 & $\mathrm{O}$ \\
\hline O13 & & & & 0.505 & 0.401 & & 1.167 & 2.07 & $\mathrm{O}$ \\
\hline O14 & 0.685 & & & & & & 1.245 & 1.93 & $\mathrm{O}$ \\
\hline O15 & & & & 0.590 & & 1.328 & & 1.92 & $\mathrm{O}$ \\
\hline O16 & 0.733 & & & & & 1.307 & & 2.04 & $\mathrm{O}$ \\
\hline O17 & 0.623 & 0.531 & & & & & & 1.15 & $\mathrm{OH}$ \\
\hline O18 & 0.628 & 0.505 & & & & & & 1.13 & $\mathrm{OH}$ \\
\hline O19 & & 0.510 & & & & & & 0.51 & $\mathrm{H}_{2} \mathrm{O}$ \\
\hline $\mathrm{O} 20$ & & 0.430 & & & & & & 0.43 & $\mathrm{H}_{2} \mathrm{O}$ \\
\hline O21 & 0.185 & $\begin{array}{l}0.460 \\
0.420\end{array}$ & & & & & & 1.07 & $\mathrm{OH}$ \\
\hline $\mathrm{O} 22$ & & & & & & & & 0.00 & $\mathrm{H}_{2} \mathrm{O}$ \\
\hline $\mathrm{O} 23$ & & & & & & & & 0.00 & $\mathrm{H}_{2} \mathrm{O}$ \\
\hline $\mathrm{O} 24$ & & & & & & & & 0.00 & $\mathrm{H}_{2} \mathrm{O}$ \\
\hline $\mathrm{O} 25$ & & & & & & & & 0.00 & $\mathrm{H}_{2} \mathrm{O}$ \\
\hline O26 & & & & & & & & 0.00 & $\mathrm{H}_{2} \mathrm{O}$ \\
\hline $\mathrm{O} 27$ & & & & & & & & 0.00 & $\mathrm{H}_{2} \mathrm{O}$ \\
\hline O28 & & & & & & & & 0.00 & $\mathrm{H}_{2} \mathrm{O}$ \\
\hline O29 & & & & & & & & 0.00 & $\mathrm{H}_{2} \mathrm{O}$ \\
\hline $\mathrm{O} 30$ & & & & & & & & 0.00 & $\mathrm{H}_{2} \mathrm{O}$ \\
\hline$\Sigma$ & 2.85 & 2.86 & 6.23 & 6.12 & 5.90 & 5.13 & 4.93 & & \\
\hline
\end{tabular}

of the bond-valence tables obtained in the present study indicates some discrepancies in the assignment of the $\mathrm{O}^{2-}$ atoms, $\mathrm{OH}^{-}$groups and $\mathrm{H}_{2} \mathrm{O}$ molecules. In their later publication about the crystal structure of upalite, Piret and Declercq (1983) reported the formula of phuralumite as $\mathrm{Al}_{2}\left[\left(\mathrm{UO}_{2}\right)_{3}\left(\mathrm{PO}_{4}\right)_{2} \mathrm{O}(\mathrm{OH})\right](\mathrm{OH})_{3}\left(\mathrm{H}_{2} \mathrm{O}\right)_{11}$. It is important to note that the current bond-valence parameters for $\mathrm{U}^{6+}$ were not available to Piret et al. (1979) and Piret and Declercq (1983), and therefore this may have contributed to their assignments of $\mathrm{O}$ atoms that we believe were incorrect in some cases.

First of all, Piret et al. (1979) reported in their bond-valence table sixteen $\mathrm{O}^{2-}$ atoms, four $\mathrm{OH}^{-}$groups and ten $\mathrm{H}_{2} \mathrm{O}$ groups, as well as the general formula $\mathrm{Al}_{2}\left[\left(\mathrm{UO}_{2}\right)_{3}\left(\mathrm{PO}_{4}\right)_{2}(\mathrm{OH})_{2}\right](\mathrm{OH})_{4}\left(\mathrm{H}_{2} \mathrm{O}\right)_{10}$, which is not in agreement with their bond-valence analysis (six $\mathrm{OH}^{-}$groups instead of four, and fourteen $\mathrm{O}^{2-}$ atoms instead of sixteen). Minerals of the phosphuranylite group are characterized by sheet of general composition $\left[\left(\mathrm{UO}_{2}\right)_{3}\left(T \mathrm{O}_{4}\right)_{2}(X)_{2}\right]$, where $T=\mathrm{P}$ or As and $X=\mathrm{O}^{2-}$ or $\mathrm{OH}^{-}$(Krivovichev and Plášil 2013). The anionic $X$ sites 


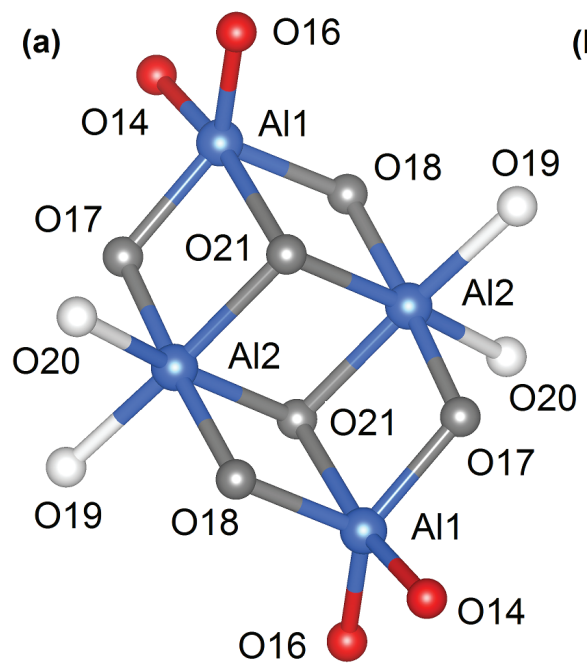

$\mathrm{Al}_{4} \mathrm{O}_{4}(\mathrm{OH})_{6}\left(\mathrm{H}_{2} \mathrm{O}\right)_{4}$

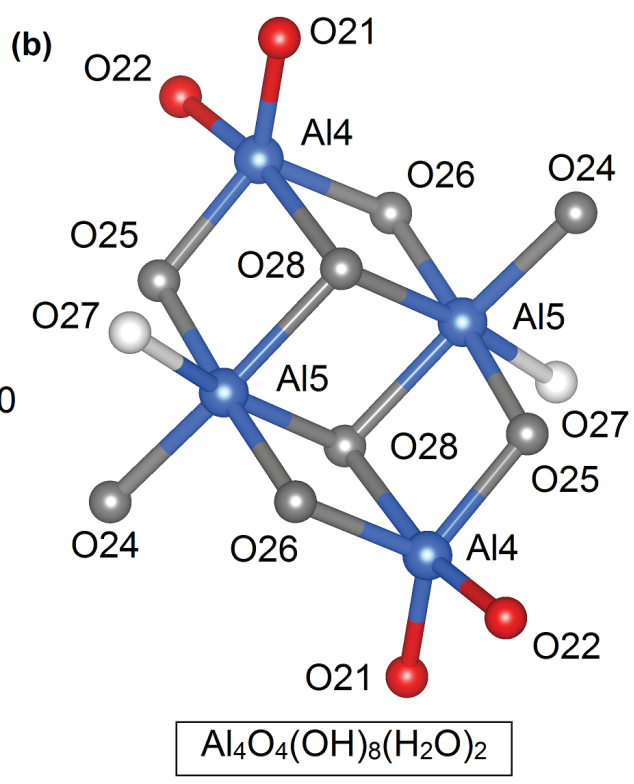

Fig. 4 Detailed view of the Al cluster occurring in the structure of phuralumite according to the present study (a) and Piret et al. (1979) (b). Oxygen atoms are red, hydroxyl groups grey and water molecules white.
(O8 and O10, this study; O14 and O17, Piret et al. 1979) have the particularity to be only connected to the uranyl polyhedra, and are occupied in the case of phuralumite by $\mathrm{O}^{2-}$ atoms (O8) and by $\mathrm{OH}$ group $(\mathrm{O} 10)$. This assignment is supported by the bond-valence sums for $\mathrm{O} 8$ and $\mathrm{OH} 10$ : 1.95 and $1.42 v . u$., respectively (v. u.: valence unit) (Tab. 5). The $\mathrm{O}-\mathrm{O}$ distances indicate that $\mathrm{O} 8$ is surrounded by $\mathrm{H}_{2} \mathrm{O} 22$ at $3.263 \AA$, limiting the interaction between these two atoms, while the OH10 is closely surrounded by $\mathrm{H}_{2} \mathrm{O} 22$ at $2.77 \AA$, indicating the presence of a $\mathrm{O} 10$ $\mathrm{H} \cdots \mathrm{H}_{2} \mathrm{O} 22$ bond (Tab. 4). Furthermore, the U-O8 bond lengths $(2.280,2.273$ and $2.238 \AA$ ), which are shorter than the U-OH10 bond lengths $(2.427,2.410$ and 2.434 A) (Tab. 3, sample VC2048), are also an indicator of a different coordination environment around the $\mathrm{O} 8$ and $\mathrm{H}_{2} \mathrm{O} 10$ atoms. It results that the sheets in the phuralumite structure have the composition $\left[\left(\mathrm{UO}_{2}\right)_{3}\left(\mathrm{PO}_{4}\right)_{2} \mathrm{O}(\mathrm{OH})\right]^{3-}$, identical to that found in upalite, $\mathrm{Al}\left[\left(\mathrm{UO}_{2}\right)_{3}\left(\mathrm{PO}_{4}\right)_{2} \mathrm{O}(\mathrm{OH})\right]$ $\left(\mathrm{H}_{2} \mathrm{O}\right)_{7}$ (Piret and Declercq 1983) and françoisite-(Nd), $\mathrm{Nd}\left[\left(\mathrm{UO}_{2}\right)_{3}\left(\mathrm{PO}_{4}\right)_{2} \mathrm{O}(\mathrm{OH})\right]\left(\mathrm{H}_{2} \mathrm{O}\right)_{6}$ (Piret et al. 1988). The distinction of the $\mathrm{O}^{2-}$ and $\mathrm{OH}^{-}$atoms located on the $X$ sites can be achieved in the same manner in the structure of upalite and françoisite-(Nd).

Taking a closer look at the bond-valence table indicates also that the $\mathrm{Al}$ atoms are coordinated by three $\mathrm{OH}^{-}$groups (O17, O18 and $\left.\mathrm{O} 21\right)$, while Piret et al. (1979) reported four $\mathrm{OH}^{-}$groups $(\mathrm{O} 24, \mathrm{O} 25, \mathrm{O} 26$ and $\mathrm{O} 28)$, despite the fact that BVS for the $\mathrm{O} 24$ is very low for hydroxyl group $(0.53 v$ v. u. $)$. Hence, the interlayer Alcluster reported in this work, $\mathrm{Al}_{4} \mathrm{O}_{4}(\mathrm{OH})_{6}\left(\mathrm{H}_{2} \mathrm{O}\right)_{4}$, differs in composition from the $\mathrm{Al}_{4} \mathrm{O}_{4}(\mathrm{OH})_{8}\left(\mathrm{H}_{2} \mathrm{O}\right)_{2}$ cluster described by Piret et al. (1979) (Fig. 4).

Finally, in all the refined structural models presented in the current work, ten of the sites assigned as $\mathrm{O}$ atoms correspond to $\mathrm{H}_{2} \mathrm{O}$ molecules, four of which have site occupancy constrained to 0.5 , given a total of eight $\mathrm{H}_{2} \mathrm{O}$ molecules per formula unit. In their structural model, Piret et al. (1979) reported ten sites occupied by water molecules; however, three of them (O35, O36 and O37) have very high thermal parameters, indicating a partial occupancy or a wrong assignment. The number of eight water molecules per unit formula stays consistent with the chemical data provided by Deliens and Piret (1979). All these new crystallographic data indicate that the formula of phuralumite, previously reported as $\mathrm{Al}_{2}\left[\left(\mathrm{UO}_{2}\right)_{3}\left(\mathrm{PO}_{4}\right)_{2}(\mathrm{OH})_{2}\right](\mathrm{OH})_{4}\left(\mathrm{H}_{2} \mathrm{O}\right)_{10}$, must be modified to $\mathrm{Al}_{2}\left[\left(\mathrm{UO}_{2}\right)_{3}\left(\mathrm{PO}_{4}\right)_{2} \mathrm{O}(\mathrm{OH})\right](\mathrm{OH})_{3}\left(\mathrm{H}_{2} \mathrm{O}\right)_{9}$.

Acknowledgements. Many thanks are due to the three anonymous reviewers for their insightful comments on the manuscript. We are grateful to Roger Warin for providing photomicrographs of the samples. We acknowledge the National Museum of Natural History of Luxembourg for the loan of specimens. FD thanks the FRS-F.N.R.S. (Belgium) for a FRIA PhD grant $n^{\circ} 93482$.

Electronic supplementary material. Supplementary crystallographic data for this paper are available online at the Journal web site (http://dx.doi.org/10.3190/jgeosci.233)

\section{References}

Agilent Technologies (2012) CrysAlis CCD and CrysAlis RED. Oxford Diffraction Ltd, Yarnton, Oxfordshire, UK

Brown ID, ALtermatT D (1985) Bond-valence parameters obtained from a systematic analysis of the inorganic crystal structure database. Acta Crystallogr B41: 244-247, with updated parameters from http://www.ccp14.ac.uk/ ccp/web-mirrors/i_d_brown/ 
BURns PC (2005) $\mathrm{U}^{6+}$ minerals and inorganic compounds: insights into an expanded structural hierarchy of crystal structures. Canad Mineral 43: 1839-1894

Burns PC, Ewing RC, Hawthorne FC (1997) The crystal chemistry of hexavalent uranium: polyhedron geometries, bond-valence parameters, and polymerization of polyhedra. Canad Mineral 35: 1551-1570

Chernikov AA (1981) Behaviour of Uranium in the Hypergene Zone. Nedra, Moscow, pp 1-207 (in Russian)

Chukanov NV, Pushcharovsky D Yu, Pasero M, Merlino S, Barinova AV, Möckel S, Pekov IV, Zadov AE, DubinchuK VT (2004) Larisaite, $\mathrm{Na}\left(\mathrm{H}_{3} \mathrm{O}\right)$ $\left(\mathrm{UO}_{2}\right)_{3}\left(\mathrm{SeO}_{3}\right)_{2} \mathrm{O}_{2} \cdot 4 \mathrm{H}_{2} \mathrm{O}$, a new uranyl selenite mineral from Repete mine, San Juan County, Utah, U.S.A. Eur J Mineral 16: 367-374

COOPER MA, HAWTHORNE FC (1995) The crystal structure of guilleminite, a hydrated $\mathrm{Ba}-\mathrm{U}-\mathrm{Se}$ sheet structure. Canad Mineral 33: 1103-1109

Cooper MA, Hawthorne FC (2001) Structure topology and hydrogen bonding in marthozite, $\mathrm{Cu}^{2+}\left[\left(\mathrm{UO}_{2}\right)_{3}\left(\mathrm{SeO}_{3}\right)_{2} \mathrm{O}_{2}\right]\left(\mathrm{H}_{2} \mathrm{O}\right)_{8}$, a comparison with guilleminite, $\mathrm{Ba}\left[\left(\mathrm{UO}_{2}\right)_{3}\left(\mathrm{SeO}_{3}\right)_{2} \mathrm{O}_{2}\right]\left(\mathrm{H}_{2} \mathrm{O}\right)_{3}$. Canad Mineral 39: 797-807

Čejka J, SeJkora J, Deliens M (1999) To the infrared spectrum of haynesite, a hydrated uranyl selenite, and its comparison with other uranyl selenites. Neu Jb Mineral, Mh 1999: 241-252

DAL Bo F (2016) Mineralogy and Crystal Chemistry of Natural and Synthetic Uranyl Oxysalts. Unpublished PhD Thesis, University of Liège, pp 1-327

Deliens M, Piret, P (1979) Les phosphates d'uranyle et d'aluminium de Kobokobo. II. La phuralumite $\mathrm{Al}_{2}\left(\mathrm{UO}_{2}\right)_{3}\left(\mathrm{PO}_{4}\right)_{2}(\mathrm{OH})_{6} \cdot 10 \mathrm{H}_{2} \mathrm{O}$ et l'upalite $\mathrm{Al}\left(\mathrm{UO}_{2}\right)_{3}\left(\mathrm{PO}_{4}\right)_{2}(\mathrm{OH})_{3}$, nouveaux minéraux. Bull Minéral 102: 333-337
FINCH RJ, MuraKami T (1999) Systematics and paragenesis of uranium minerals. In: BuRns PC, FINCH RJ (eds) Uranium: Mineralogy, Geochemistry and the Environment. Mineralogical Society of America and Geochemical Society Reviews in Mineralogy and Geochemistry 38: pp 91-180

Krivovichev SV, PlášIl J (2013) Mineralogy and crystallography of uranium. In: BuRns PC, Sigmon GE (eds) Uranium: From Cradle to Grave. Mineralogical Association of Canada Short Courses 43: pp 15-119

Momma K, IzUmi F (2011) VESTA 3 for three-dimensional visualization of crystal, volumetric and morphology data. J Appl Crystallogr 44: 1272-1276

PIRET P, DeCLERCQ J-P (1983) Structure cristalline de l'upalite $\mathrm{Al}\left[\left(\mathrm{UO}_{2}\right)_{3} \mathrm{O}(\mathrm{OH})\left(\mathrm{PO}_{4}\right)_{2}\right] \cdot 7 \mathrm{H}_{2} \mathrm{O}$. Un exemple de macle mimétique. Bull Minéral 106: 383-389

Piret P, Piret-Meunier J, DeclercQ J-P (1979) Structure of phuralumite. Acta Crystallogr B35: 1880-1882

Piret P, Deliens M, Piret-Meunier J (1988) La françoisite(Nd), nouveau phosphate d'uranyle et de terres rares; propriétés et structure cristalline. Bull Minéral 111: 443-449

PLÁŠIL J (2014) Oxidation-hydration weathering of uraninite: the current state-of-knowledge. J Geosci 59: 99-114

Plášil J, SEJKora J, ČEJKa J, ŠKoda R, Goliáš V (2009) Supergene mineralization of the Medvědín uranium deposit, Krkonoše Mountains, Czech Republic. J Geosci 54: $15-56$

SHELDRICK GM (2008) A short history of SHELX. Acta Crystallogr A64: 112-122

Vochten R, Blaton N, Peeters O, Deliens M (1996) Piretite, $\mathrm{Ca}\left(\mathrm{UO}_{2}\right)_{3}\left(\mathrm{SeO}_{3}\right)_{2}(\mathrm{OH})_{4} \cdot 4 \mathrm{H}_{2} \mathrm{O}$, a new calcium uranyl selenite from Shinkolobwe, Shaba, Zaire. Canad Mineral 34: 1317-1322

WiLson AJC (1992) International Tables for X-ray Crystallography, vol. C. Kluwer Academic Press, London, pp 1-883 
